\title{
Leading baryon production in ep collisions
}

\section{Lorenzo Rinaldi* ${ }^{\dagger}$}

DESY, Notkestrasse 85, D-22607 Hamburg, Germany

E-mail: rinaldi@mail.desy.de

The production of leading protons and leading neutrons has been studied at HERA with the detectors $\mathrm{H} 1$ and ZEUS. The leading proton production cross sections in DIS reactions have been measured as a function of the longitudinal fractional momentum $x_{L}$ and transverse squared momentum $p_{T}^{2}$ and compared to theoretical models. The longitudinal and transverse leading neutron spectra have been measured both in DIS and photoproduction and the distributions are compared to the predictions of pion-exchange models, also incorporating absorption effects. The production of events with two hadronic jets and a leading neutron has been measured and the comparison between $\mathrm{H} 1$ and ZEUS results are shown.

Diffraction 06, International Workshop on Diffraction in High-Energy Physics September 5-10, 2006

Adamantas, Milos island, Greece

\footnotetext{
*Speaker.

$\dagger$ On behalf of $\mathrm{H} 1$ and ZEUS Collaborations.
} 


\section{Introduction}

In $e^{ \pm} p$ scattering at HERA, a significant fraction of events contains a baryon carrying a large fraction of the incoming proton energy [1] . The production mechanism of these low-transversemomentum leading baryons is not yet completely understood. The leading baryons can result from the fragmentation of the proton remnant, but their production can be explained also in terms of an exchanged object, according to the Regge theory. It has been observed that the leading baryons can rescatter on the hadronic components of the photon: these absorptive effect are taken into account in some theorethical models [2, 3].

The leading proton cross sections in DIS have been measured as a function of the leading proton variables $x_{L}$ and $p_{T}^{2}$ and the results have been compared with a theoretical model.

The leading neutron cross sections and $b$-slopes have been measured in DIS and photoproduction and they have been compared to check the absorption and rescattering effects. Also the dijet photoproduction events with a leading neutron have been studied. Finally all the results have then been compared to theoretical models.

\section{Experimental setup}

A detailed description of the H1 and ZEUS detectors can be found elsewhere [

The leading baryon detectors were positioned in the forward direction, along the proton beamline (Fig. 1). In the ZEUS detector, the leading protons were detected using the Leading Proton Spectrometer (LPS). It consisted of 36 planes of silicon microstrip detectors grouped in six stations, $\mathrm{S} 1$ to $\mathrm{S} 6$, positioned between $z=20 \mathrm{~m}$ and $z=90 \mathrm{~m}$ from the interaction point. The stations were inserted near the proton beam and detected the charged particles that were deflected by the proton beamline magnetic elements. The resolution was better than $1 \%$ in longitudinal momentum and of $5 \mathrm{MeV}$ in transverse momentum; the latter was dominated by the beam-emittance in the transverse plane $(40-90 \mathrm{MeV})$.

The ZEUS Forward Neutron Calorimeter (FNC) was positioned at $z=106 \mathrm{~m}$ from the interaction point after the LPS. It was a lead-scintillator calorimeter with an energy resolution $\sigma_{E} / E=$ $0.70 / \sqrt{E(G e V)}$. Three planes of veto counters were located in front of the FNC and used to veto charged particles. The Forward Neutron Tracker (FNT) was a hodoscope installed in the FNC at a depth of one interation length to measure the position of neutron showers. The geometrical acceptance of the detector limited neutron scattering angles to smaller than $\theta_{n} \sim 0.75 \mathrm{mrad}$ with approximately $30 \%$ azimuthal coverage.

The H1 Forward Neutron Calorimeter consisted of interleaved layer of $2 \mathrm{~m}$ long lead strips and scintillator fibres and it was located at $107 \mathrm{~m}$ from the interaction point. The energy resolution is $\sigma_{E} / E \approx 20 \%$. Two segmented planes of hodoscopes situated in front of the FNC were used to veto charged particles. The neutron detection efficiency was $(93 \pm 5) \%$ and the acceptance was limited to neutron scattering angles of $\theta_{n} \sim 0.8 \mathrm{rad}$.

\section{Event selection}

The leading proton data were collected using the ZEUS detector during 1996-97 $e^{+} p$ data taking period, corresponding to an integrated luminosity of $12.8 \mathrm{pb}^{-1}$. For the DIS selection, the 


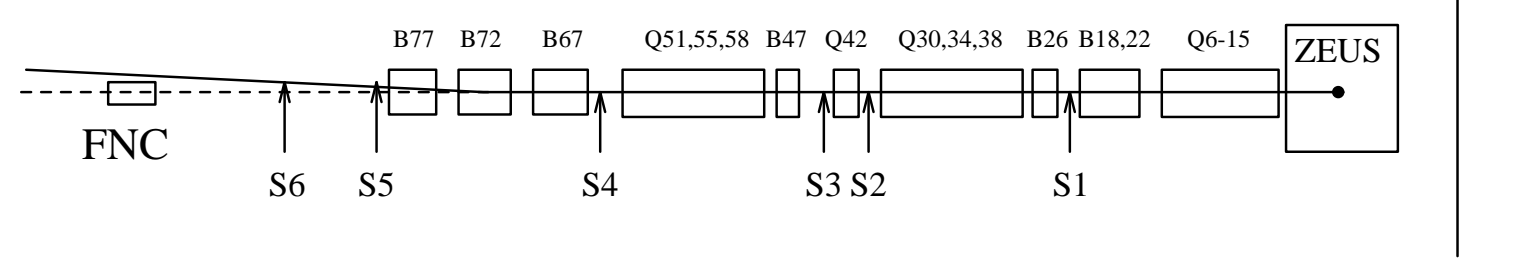

Figure 1: The proton beamline downstream from the ZEUS interaction region.

events were required to have the exchanged-photon virtuality $Q^{2}>3 \mathrm{GeV}^{2}$ and the invariant mass of the hadronic system $45<W<225 \mathrm{GeV}$. A good track was required in the LPS having $x_{L}>0.56$ and $p_{T}^{2}<0.5 \mathrm{GeV}^{2}$. A further set of cuts was applied to enhance the DIS selection and to suppress the background.

To determine the acceptance of the apparatus, DIS events were generated using DJANGO [6]. This sample included diffractive events, produced in the generator using the soft-colour-interaction mechanism. To describe the data, it was necessary to reweight the leading proton $x_{L}$ and $p_{T}^{2}$ distributions generated by the Monte Carlo.

The events with a leading neutron were selected during $2000 e^{+} p$ data taking period, requiring at least $165 \mathrm{GeV}$ of energy to be deposited in the FNC. Offline cuts were used to select clean neutron candidates, and further cuts were applied to select neutrons with a well reconstructed position.

The DIS events were collected using a trigger that detected the scattered positron in the ZEUS calorimeter, limiting the acceptance to the kinematic range $Q^{2}>2 \mathrm{GeV}^{2}$ with mean photon virtuality of $\left\langle Q^{2}\right\rangle \simeq 14 \mathrm{GeV}^{2}$.

The inclusive photoproduction sample of $6 \mathrm{pb}^{-1}$ was collected using a trigger that required at least $5 \mathrm{GeV}$ in a positron tagger in coincidence with $464 \mathrm{MeV}$ in the rear calorimeter. The acceptance of the positron tagger limited the photon virtuality to $Q^{2}<0.02 \mathrm{GeV}^{2}$.

In the ZEUS dijet photoproduction sample, at least two jets were reconstructed offline with the $k_{T}$ algorithm, satisfying the conditions: $-1.5<\eta^{\text {jet }}<2.5$ and $E_{T}^{\text {jet } 1}>7.5 \mathrm{GeV}$ and $E_{T}^{\text {jet } 2}>6.5$ $\mathrm{GeV}, 130<W<280 \mathrm{GeV}$ and no positron detected in the calorimeter. The integrated luminosity of the DIS and photoproduction dijet samples was $40 \mathrm{pb}^{-1}$.

The $\mathrm{H} 1$ dijet sample selection was carried out in a similar way, by requiring two jets reconstructed with the cone algorithm with $E_{T}^{\text {jet } 1}>7 \mathrm{GeV}$ and $E_{T}^{\text {jet } 2}>6 \mathrm{GeV}$ in the pseudorapidity range $-1<\eta^{j e t}<2$.

\section{Models}

The one-pion-exchange model [7] is usually applied to describe leading neutron production for large $x_{L}$. In this model the cross section for the semi-inclusive reaction $e p \rightarrow e X n$ factorizes into two terms, the flux of virtual pions emitted by the proton and the cross section of the $\gamma^{*} \pi$ interaction, $\frac{d^{2} \sigma}{d x_{L} d t}=f_{\pi / p}\left(x_{L}, t\right) \sigma_{\gamma^{*} \pi}$, where $t$ is the virtuality of the exchanged pion. However this vertex factorization can be violated by neutron absorption [2, 3], which is the rescattering of the neutron on the photon hadronic component. At large photon size (namely small values of the photon virtu- 


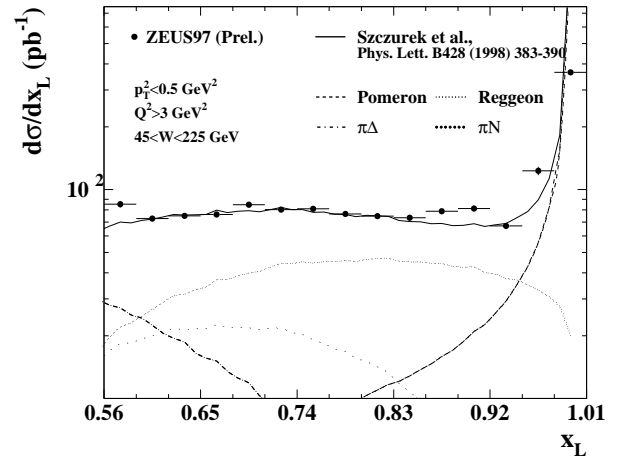

Figure 2: Leading proton differential cross section as a function of $x_{L}$. The curves are from a Reggeon-exchange model [8].

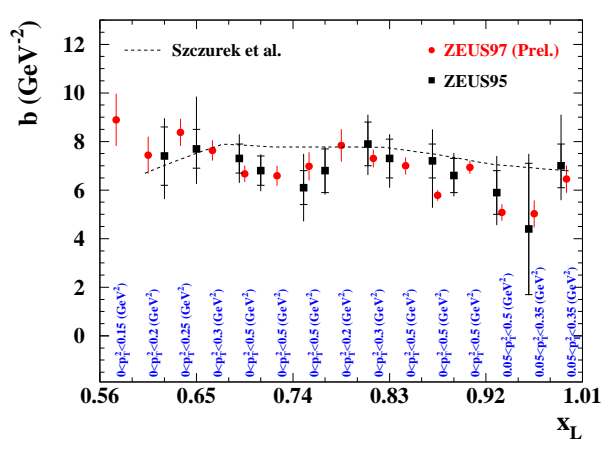

Figure 3: Leading proton $b$-slopes as a function of $x_{L}$ for two different sets of data. The curve is from a Reggeon-exchange model [8].

ality $Q^{2}$ ) or small $n-\pi$ sizes (large neutron $p_{T}$ 's) more absoprtion is expected. Other factorization violation effects can be induced by the energy redistribution due to the neutron migration in the phase space. There may also be additional particle exchanges such as $\rho$ and $a_{2}$.

\section{Results}

The leading proton cross section $d \sigma_{e p \rightarrow e p X} / d x_{L}$ measured in the kinematic range $x_{L}>0.56$, $p_{T}^{2}<0.5 \mathrm{GeV}^{2}, Q^{2}>3 \mathrm{GeV}^{2}$ and $45<W<225 \mathrm{GeV}$ is shown in Fig. 2 It is found to be flat up to the diffractive peak. In the figure is also shown the prediction of a Reggeon-exchange based model [8] which agrees with the experimental measurements. The double differential cross section $d^{2} \sigma_{e p \rightarrow e p X} / d x_{L} d p_{T}^{2}$ as a function of $p_{T}^{2}$ in bins of $x_{L}$ was measured and fitted to the exponential function $A \cdot e^{-b \cdot p_{T}^{2}}$. The $b$-slopes as a function of $x_{L}$ are shown in Fig. B. There is no evidence for an $x_{L}$ dependence of the $p_{T}^{2}$ slope and the observed fluctuations may be ascribed to the different $p_{T}^{2}$ fit ranges. In Fig. 3 is also shown the prediction from Szuzureck et al. and it is found to be in agreement with the measurements.

Fig. 4 shows the normalized differential cross section $\left(1 / d \sigma_{D I S}\right) d \sigma_{e p \rightarrow e X n} / d x_{L}$ for neutrons in DIS with scattering angles $\theta_{n}<0.75 \mathrm{mrad}$, corresponding to the kinematic range $p_{T}^{2}<0.476 x_{L}^{2}$ $\mathrm{GeV}^{2}$. It falls to zero at the endpoint $x_{L}=1$, reaches a maximum near $x_{L}=0.7$ and falls as $x_{L}$ decreases due to the vanishing region of $p_{T}$ space. The curves correspond to recent predictions from a one-pion-exchange model incorporating the effects of neutron absorption and energy redistribution [3].

The ratio $\rho\left(x_{L}\right)$ between the normalized differential distribution in photoproduction and DIS for scattering angle cut $\theta_{n}<0.75 \mathrm{mrad}$ is shown in Fig. F. In the range $0.2<x_{L}<0.55$, the ratio drops slightly but rises for higher $x_{L}$ values, exceeding unity for $x_{L}>0.9$. The dashed curve is the expectation for the suppression of leading neutrons in photoproduction relative to DIS from a model of absorption [2]. The $\gamma p$ interactions have a power law dependence $\sigma \propto W^{2 \lambda}$, with different values of $\lambda$ for DIS and photoproduction. This results in a dependence on the ratio that is proportional to $\left(1-x_{L}\right)^{\Delta \lambda}$, with $\Delta \lambda \approx-0.1$. Applying this to the absorption suppression factor results in the solid curve in Fig. 5, which is observed to be in good agreement with the data. 


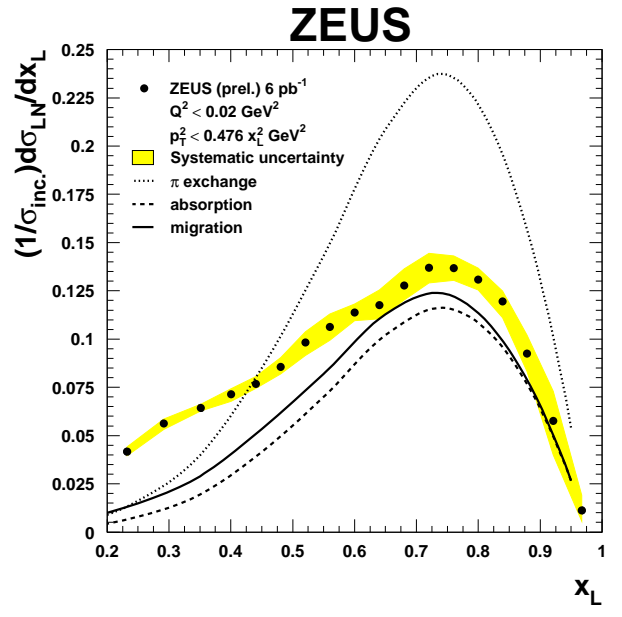

Figure 4: Leading neutron energy spectra for the DIS sample. Curves are from the KKMR model [3].

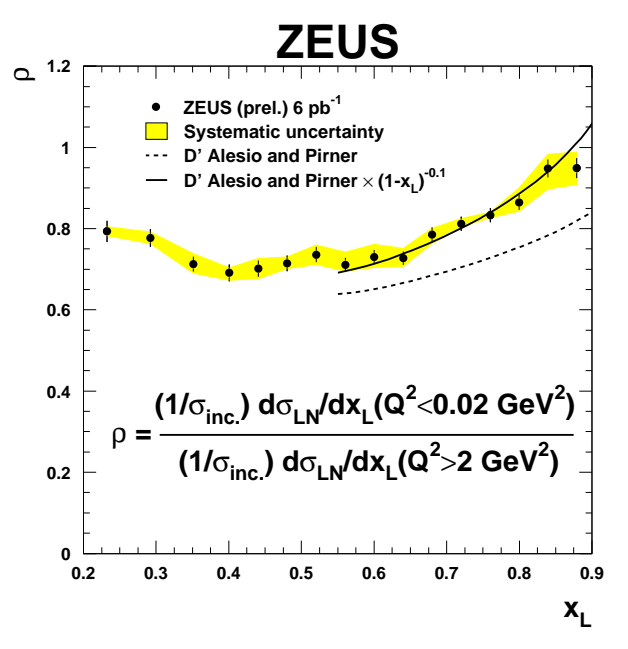

Figure 5: Leading neutron ratio of the $x_{L}$ distributions photoproduction over DIS. The curves are the prediction of a rescattering model [2].

The $p_{T}^{2}$ distributions for the DIS and photoproduction samples, normalized to unity at $p_{T}^{2}=0$, are shown in Fig. 6. The data are well described by a parametrization of the form $A \cdot e^{-b \cdot p_{T}^{2}}$, shown as the superimposed curves. The photoproduction distributions are clearly steeper in the range $0.6<x_{L}<0.9$. The difference of the slopes $\Delta b=b\left(Q^{2}<0.02 \mathrm{GeV}^{2}\right)-b\left(Q^{2}>2 \mathrm{GeV}^{2}\right)$ is less sensitive to systematic effects than each of the individual slopes. These values are shown in Fig. 7 . Within the systematic uncertainties the slopes for photoproduction are clearly larger in the range $0.6<x_{L}<0.9$, with $\Delta b=0.5-1.0 \mathrm{GeV}^{-2}$. The depletion of neutrons at large $p_{T}^{2}$ is qualitatively consistent with the expectations from absorption models.

The leading neutron $b$-slopes as a function of $x_{L}$ are shown in Figs. 8 and 9 . In Fig. 8 the slopes are compared to leading proton data. The different shapes of the distributions can be attributed to the different exchanges dominating leading neutron and leading proton production. At $x_{L} \sim 0.6-$ 0.7 , both measurements agree, suggesting that the amount of pion exchange in the two processes is similar. In Fig. 9 the leading neutron slopes are compared to a pion-exchange-model including additional particles exchanges [3], which gives a good description of the experimental data.

The ratios of the dijet cross sections with and without the requirement of a leading neutron measured by $\mathrm{H} 1$ and ZEUS are shown in the Figs. 10 and 11 . The ratios were plotted as a function of the transverse energy $E_{T}^{j e t}$, the pseudorapidity $\eta^{j e t}$ of the jets and $x_{\gamma}$, the fraction of the photon momentum participating to the hard interaction. At low $x_{\gamma}$, the photon fluctuates into a $q \bar{q}$ state and rescattering on the leading neutron can occur and, in principle, a lower fraction of dijet events tagged with a leading neutron is expected. The experimental measurements are characterized by large uncertainties, also related to the MC simulations (shown in the plots) that were used for the calculation of the acceptance, and thus we can not conclude that, in this case, there is a strong evidence of a vertex factorization violation. 


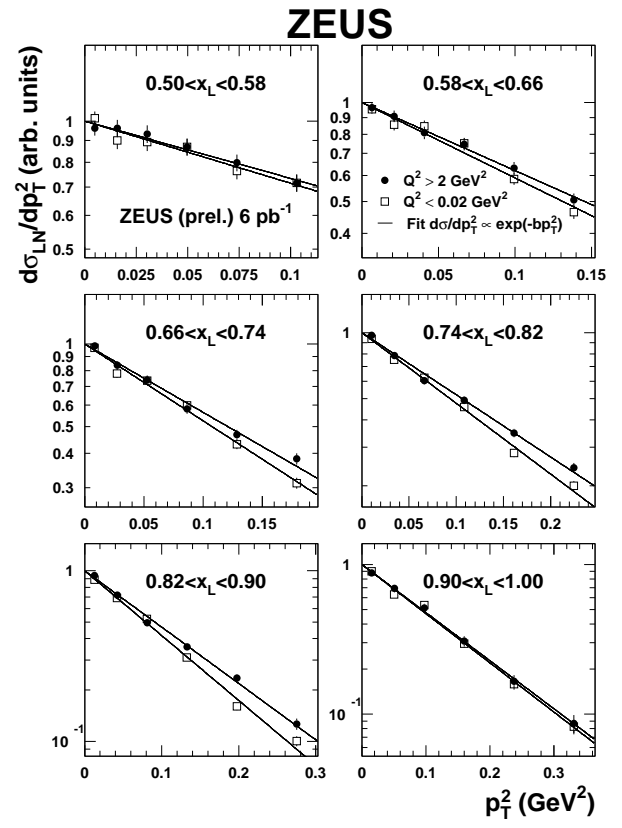

Figure 6: Leading neutron $p_{T}^{2}$ distributions for photoproduction and DIS in bins of $x_{L}$, normalized to unity at $p_{T}^{2}=0 \mathrm{GeV}^{2}$.

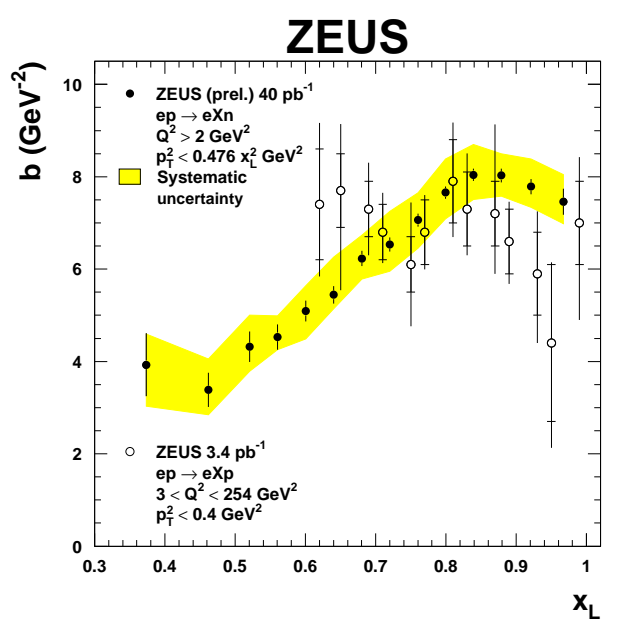

Figure 8: Leading neutron DIS slopes compared to leading proton DIS slopes.

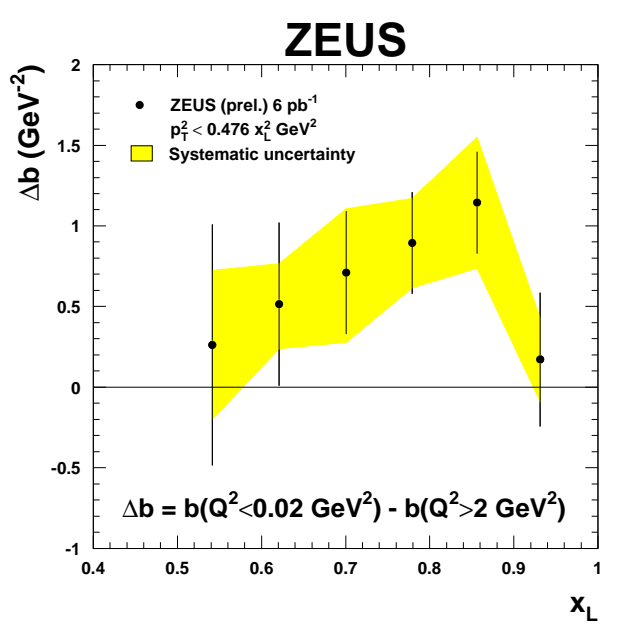

Figure 7: Leading neutron difference between the slopes for photoproduction and DIS.

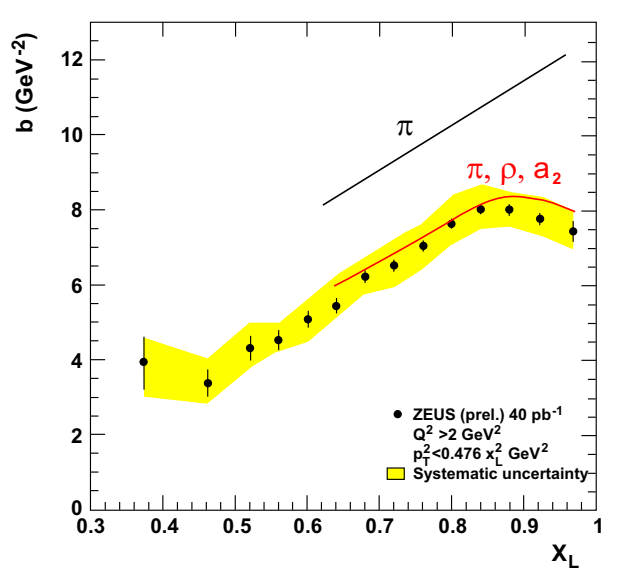

Figure 9: Leading neutron DIS slopes as a function of $x_{L}$ compared to predictions from KKMR model [3].

\section{References}

[1] ZEUS Coll., M. Derrick et al., Phys. Lett. B384 (1995) 388.

H1 Coll, C. Adloff et al., Eur. Phys. J. C6 (1999) 587.

H1 Coll, C. Adloff et al., Nucl. Phys. B619 (2001) 3.

ZEUS Coll., J. Breitweg et al., Nucl. Phys. B569 (2001) 3.

ZEUS Coll., J. Breitweg et al., Nucl. Phys. B637 (2002) 3.

ZEUS Coll., J. Breitweg et al., Nucl. Phys. B658 (2003) 3.

H1 Coll., A. Aktas et al., Eur. Phys. J. C41 (2005) 273. 

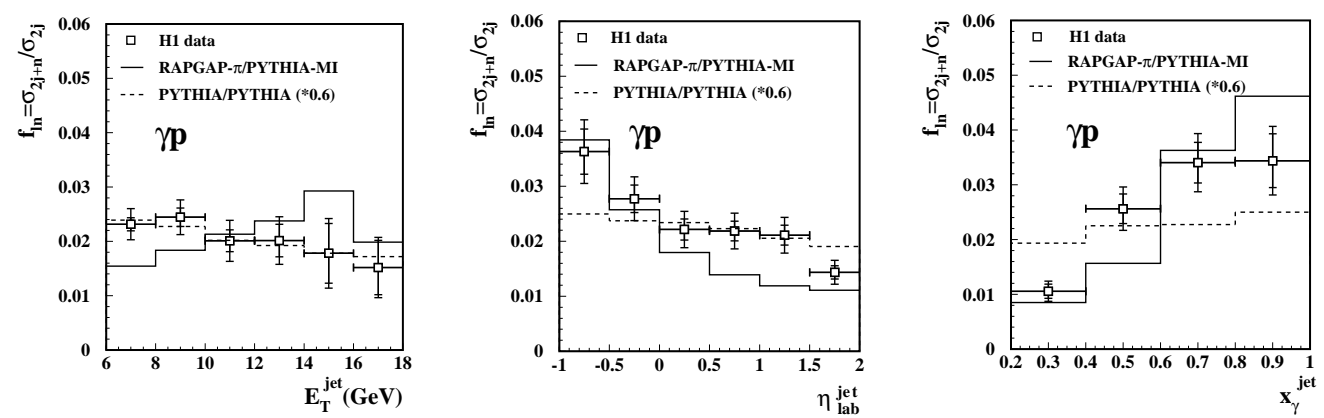

Figure 10: H1 data: Ratio of the cross section for dijet photoproduction with a leading neutron to inclusive dijet photoproduction, as a function of $E_{T}^{j e t}, \eta^{j e t}$ and $x_{\gamma}$. The curves are the MC predictions.
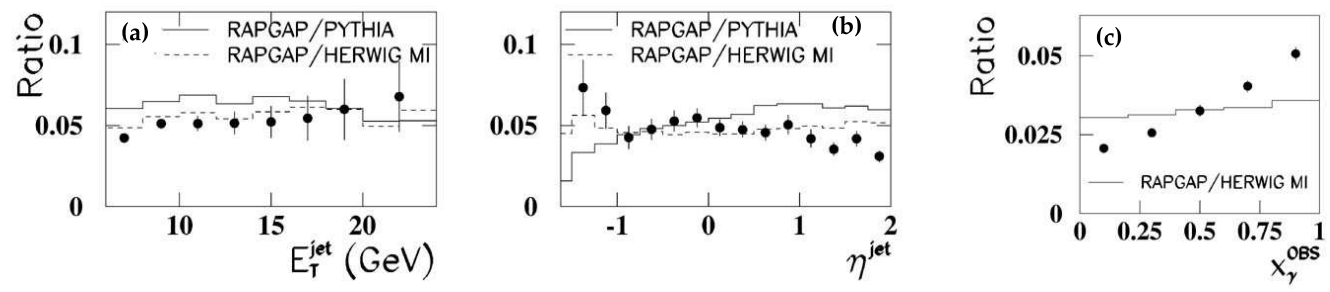

Figure 11: ZEUS data: Ratio of the cross section for dijet photoproduction with a leading neutron to inclusive dijet photoproduction, as a function of $E_{T}^{\text {jet }}(\mathrm{a}), \eta^{\text {jet }}$ (b) and $x_{\gamma}$ (c). The curves are the MC predictions.

[2] U. D’Alesio and H.J. Pirner, Eur. Phys. J. A7 (2000) 109.

[3] A.B. Kaidalov, V.A. Khoze, A.D. Martin and M.G. Ryskin, Preprint IPP-06-10, DCPT-06-20 (2006) [hep-ph/0602215].

V.A. Khoze, A.D. Martin and M.G. Ryskin, Preprint IPP-06-36, DCPT-06-72 (2006)

[hep-ph/0606213].

[4] H1 Coll., I. Abt et al., Nucl. Intrum. Methods A386 (1997) 310.

H1 Coll., I. Abt et al., Nucl. Intrum. Methods A386 (1997) 348.

[5] ZEUS Coll. U. Holm (ed.), The ZEUS Detector. Status Report (unpublished), DESY (1993), available on http://www-zeus.desy.de/vluebook/bluebook.html.

[6] K. Charchuła, G.A. Schuler and H. Spiesberger, Comput. Phys. Commun. 81 (1994) 381.

[7] M. Bishari, Phys. Lett. B38 (1972) 510.

L.L. Frankfurt, L. Mankiewicz and A. Szczurek, Z. Phys. A334 (1989) 343.

K. Golec Biernat, J. Kwiecinski and A. Szczurek, Phys. Rev. D56 (1997) 3955.

[8] A. Szczurek, N.N. Nikolaev and J. Speth, Phys. Lett. B428 (1998) 383. 